\title{
Hybrid Cooling System for Solar PV Panel
}

\author{
Rickric O. Gratela, Joyce Ann S. Martes, Gerome I. Pagatpatan, Edmon O. Fernandez, Timothy M. \\ Amado, Ira C. Valenzuela* \\ Department of Electronics Engineering, Technological University of the Philippines, Manila, Philippines \\ *ira_valenzuela@tup.edu.ph
}

\begin{abstract}
One convenient technology used nowadays are solar photovoltaic panels. It is a device used to alter light energy to electrical energy. Most of the panels have low energy conversion resulting to low efficiency of the panel itself. Among the different factors, temperature mostly affects the process of conversion which causes deficiency throughout the operating period on the long run. As solution, varieties of different cooling systems were developed to sustain the operating temperature in order to not go over a specific limit. This study compares the solar photovoltaic panel power output without cooling system and with the hybrid air- and water-cooling fluids applied to the system simultaneously. The angle is fixed at 15 degrees based on the country's latitudinal location where the experiments were performed. Current, voltage, power, irradiance and efficiency were gathered data using each parameter sensor. Results shows a substantial rise $f$ efficiency on the panel that uses a hybrid cooling system liken to the standard power rating of the panel used.
\end{abstract}

Key words : Air Cooling, Water Cooling, PV System, Solar Panel Efficiency

\section{INTRODUCTION}

Different factors affect the generated output power and life span of a photovoltaic module. Influenced by many factors such as material degradation, solar irradiance, temperature of the module, parasitic resistances, fill-factor, PID, tilt-angle, etc. Above all, the temperature is the most dominant. A study shows that overall, the temperature reduces the power output from $0.4 \%$ to $0.5 \%$ rate per degree of temperature increase[1].

It is now a challenge to increase the efficiency of the PV panel to be more reliable and more effective. There is entitled Automatic Sun Tracking System (ASTS) which uses the sun as a guiding source instead of using the earth as its reference to keep the panel in front of the visible sun[2]. More researches were performed such as one focused in improving the PV system efficiency using a concentrator and a water cooling system (CPV), a hybrid water cooled PV system which is incorporated with a heat exchanger on the rearmost panel surface that significantly affected the module temperature to fall around $20 \%$ causing to an increased efficiency of $9 \%$ and, a hybrid photovoltaic/thermal (PV/T) system where results have shown the adverse effect of the operating temperature to the performance of the pv cells[3]. It is also important to consider the electrical characteristics of the photovoltaic module. This is where Standard Test Conditions appear. It includes the intensity/power of sunlight, the air mass or the thickness of air that the sunlight passes through before reaching the module, and the cell temperature as stated, is different from ambient air [4].

Hybrid is the term for air- and water-cooling fluids, as used in this study aims to decrease the deficiency caused by the increase of operating temperature. Both the cooling method were optimized to activate simultaneously after reaching over 35degreesCelsius of temperature. In line with this, the PV panel was installed on a solar panel rack at a fixed angle of 15 degrees with respect to its geographical location.

This study provides data which can be used to ascertain the adequate cooling system in accordance to their desired category and expenses to consider. Likewise, owners of solar PV panels will benefit as well as those who intend to utilize these panels as source of electricity both for household and for commercial use.

This paper is organized as follows: Section II contains the different approaches of cooling systems for solar PV panels, and Section III discusses the study's methodology of the cooling system and testing properties. The development discussion and result are contained in Section IV while Section V holds the research conclusion.

\section{RELATED WORKS}

Grubisic-Cabo et al. discussed two main types of cooling technique namely, the active or cooling by consuming energy like pumps and fans, and the passive cooling by using natural convection/conduction for heat extraction. Passive cooling can either be through air, water or conduction. Among these, the water passive cooling was proven more efficient by maintaining a 30 degrees Celsius temperature, the efficiency increased by $20 \%$. Active cooling has two most common methods: based on water and based on air. Either a pump or a fan is needed to maintain the fluid circulation by consuming power to cool the PV panel[5].

Nizetic et al. introduced an active cooling technique by using water to spray on the panel's surface. Both sides of the panel 
were installed by water spray that activates simultaneously. Results shows that there has been a maximal overall increase of 16.3 percent in the electric power output and 14.1 percent with its electrical efficiency[6].

Tolentino et al. studied the use of ripple correlation control algorithm (RCC) for an analog maximum power point tracking (MPPT) controller integrated circuit which also involves enhancing of efficiency by improving the operating temperature. Results of simulation presented a tracking efficiency of $97.42 \%$ at $1000 \mathrm{~W} / \mathrm{m}^{2}$ solar irradiance with 25 degrees Celsius of temperature[7].

Yusoff et al. conducted a research using a solar simulator while implementing active air-cooling method for the solar PV panel. The study compares the operating temperature of the PV panel with and without air cooling mechanism. The setup was running at high operating temperature due to high solar radiation. These confirms that as the temperature during operation increases, the more negatively it affects the electrical efficiency of the panel[8].

\section{METHODOLOGY}

\subsection{Cooling System}

Complexity of the system comes with costs. This study used a cost-efficient approach with the intention of providing a higher efficient system. The higher the power rating of the solar panel is, the more it retorts in its physical dimension[1]. Thus, it affects the size, power and number of the components needed to cool down the panel's temperature. Hence, the system was assembled in consideration of the solar panel in deliberation of its efficiency: an output that should be highly sufficient to supply the water pump, the fan, and the DC load provided.

Four (4) fans were installed to the two panel setups at the rear side while the front topmost part was installed with eight (8) nozzles evenly spaced into 2 nozzles per square module to cover the whole panel.

The setup proposed has a frame with an adjustable stand and angle brackets which made it convenient to relocating since it can be took apart. The frame must be set to be at an angle $15^{\circ}$ from the horizontal but may set at most $\pm 5 \%$ allowance as shown in Figure 1. Aclay pot was used as storage in consideration to the recycling water process compared to using a conductive and convective water container since its exposure will sooner or later affect the stored water's temperature[9]. It helped in preserving the low temperature of the stored liquid.

A water pump was used to install the water-cooling system. Nozzles were set at the topmost part of the panel having a fixed flow rate of 2 liters per minute. On top of the panel's size, this flow rate is just right the amount to entirely cover the module. A study that used a $2 \mathrm{~L} / \mathrm{min}$ water flow rate without other use of cooling devices yielded $62 \mathrm{Wh}$. As a result, the output energy was increased to $77 \mathrm{Wh}$, a $24 \%$ total gain[10].
An inlet configuration decreases the PV panel temperature more effectively than using an outlet configuration. The photovoltaic panel used has an actual dimension of 58 by 26 by 1.3 inches. Concerning the surface area of the panel to be catered, four fans were installed at back almost similar to the study using 4 units of fan that has decreased an average temperature of 51.31-35.28 degrees Celsius and has the power output increased to $44.34 \%$. Fans used in this study has dimensions of 5.6 by 5.3 by 1.9 inches. Four fans were installed in consideration for the power consumption of the air-cooling operation[11].

Figure 1 shows the dimensions of the proposed setup for the system. Measurements are observed so that the air cooling will be able to cater the back of a 150-Watt panel[12].

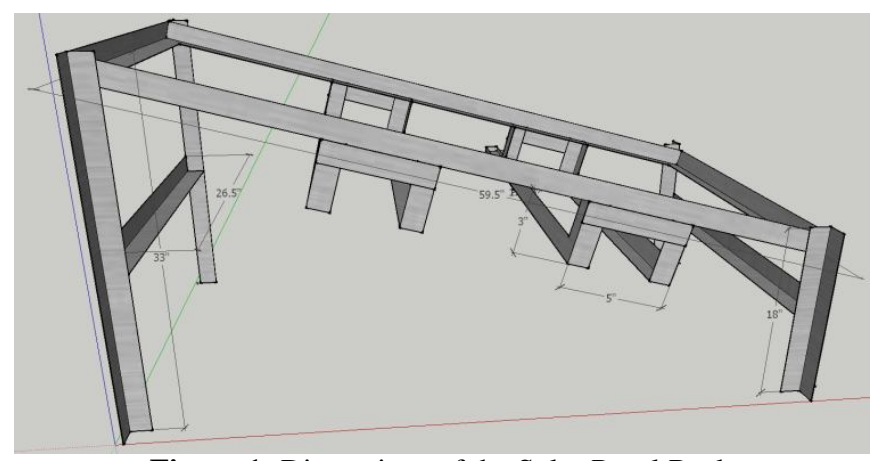

Figure 1: Dimensions of the Solar Panel Rack

Table 1 lists the solar panel specifications chosen and considered in promoting the hybrid cooling system. Since the system is intended to do self-cleaning method through the water spray on its surface, it also signifies that not only the liquid is being recycled but also the energy. The hybrid cooling system will also use the solar panel as its energy source.

Table 1: Solar Panel Specifications

\begin{tabular}{|l|l|}
\hline \multicolumn{2}{|c|}{ Electrical Characteristics } \\
\hline Rated Maximum Power (Pmax) & $150 \mathrm{~W}$ \\
\hline Power Tolerance Range & $0-+5 \mathrm{~W}$ \\
\hline Open Circuit Voltage (Voc) & $22.7 \mathrm{~V}$ \\
\hline Peak Voltage (Vmp) & $18.4 \mathrm{~V}$ \\
\hline Short Circuit Current (Isc) & $8.58 \mathrm{~A}$ \\
\hline Peak Current (Imp) & $8.16 \mathrm{~A}$ \\
\hline Max. System Voltage & $1000 \mathrm{~V}$ \\
\hline Max. Series Fuse Rating & $15 \mathrm{~A}$ \\
\hline
\end{tabular}

\subsection{Testing and Experimentations}

Figure 2 shows the setup without cooling system and with cooling system. The testing was conducted to assess the capability of the cooling system to significantly raise the efficiency of the panel by producing a larger amount of power 
while subsiding heat to lower temperature. The data presents an increase of $18.27 \%$ in efficiency by just installing the cooling system alone. Figures 3 and 4 shows the nozzle installation and the clay pot used for the cooling system.

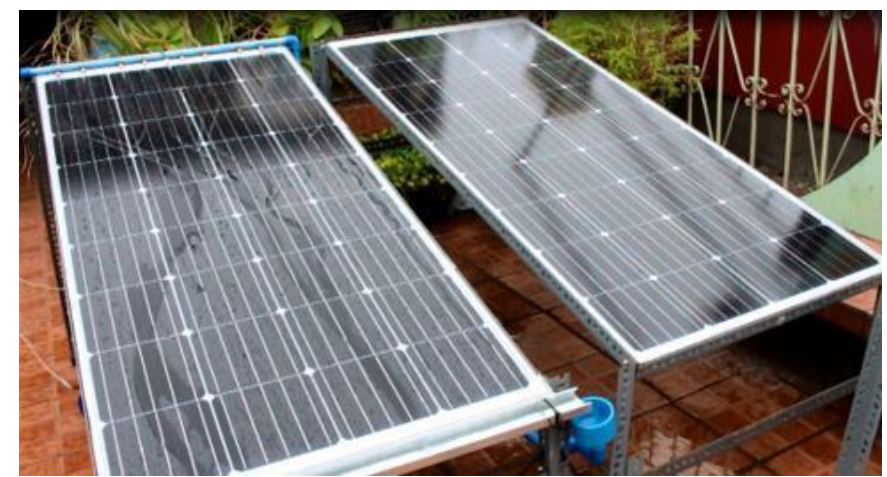

Figure 2: Setup with (left) and without (right) Cooling System

Figure 2a shows the placed cooling system nozzles which is spaced evenly on the topmost part of the panel while figure $2 b$ shows the clay pot used as water container for water storage.

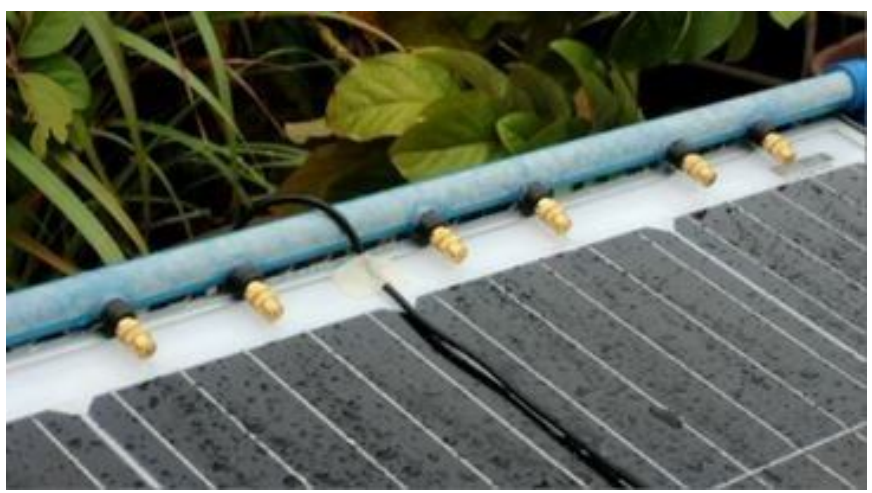

(2a)

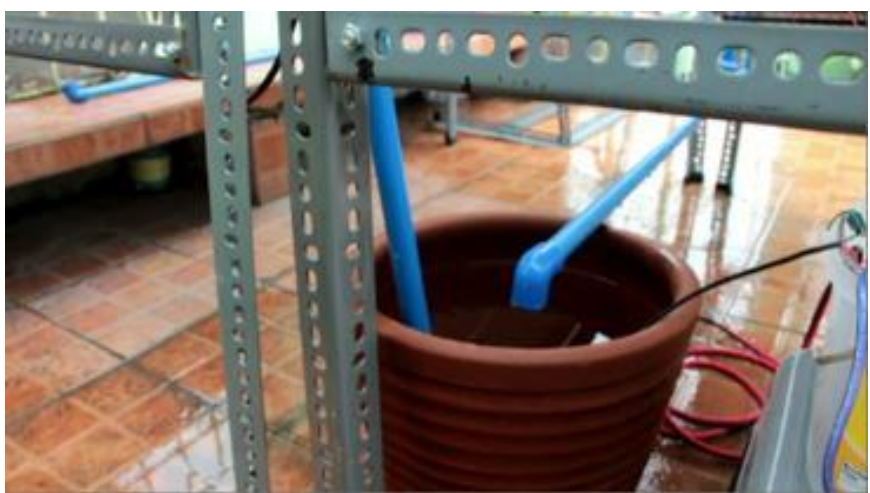

(2b)

\section{RESULTS AND DISCUSSIONS}

Initially, raw data of the PV panel parameters: temperature, voltage, current, irradiance were extracted from the data logger. These data were obtained from testing and experimentation of the panel with and without cooling system. The specifications of the panel from Table 1 were verified and were compared to the output data of the panel

with the hybrid cooling system applied.Both tests runsimultaneously from 10:00 in the morning to 5:00 in the afternoon.

Tables below gives the comparison of the first setup of a solar PV panel alone and the other setup with the hybrid cooling system. Even though taken at the same time, the difference of the voltages of the two are close while the temperature, the current, as well as the power has noticeably high difference. Purely, the temperature measured is the actual temperature of the panel.

Table 2: Output Data of Sole PV Panel

\begin{tabular}{|c|r|r|r|c|}
\hline Time & \multicolumn{1}{|c|}{ Temp } & Voltage & Current & Power \\
\hline $10: 00$ & 41.63 & 22.09 & 0.44 & 9.76 \\
\hline $10: 15$ & 44.38 & 21.82 & 0.40 & 8.66 \\
\hline $10: 30$ & 42.69 & 20.62 & 0.38 & 7.73 \\
\hline $10: 45$ & 44.88 & 21.64 & 0.36 & 7.70 \\
\hline $11: 00$ & 47.06 & 21.08 & 0.35 & 7.42 \\
\hline $11: 15$ & 45.56 & 20.82 & 0.28 & 5.75 \\
\hline $11: 30$ & 47.38 & 21.10 & 0.37 & 7.76 \\
\hline $11: 45$ & 49.13 & 20.99 & 0.47 & 9.91 \\
\hline $12: 00$ & 46.63 & 20.45 & 0.45 & 9.26 \\
\hline $12: 15$ & 50.75 & 20.03 & 0.37 & 7.47 \\
\hline $12: 30$ & 53.38 & 20.55 & 0.43 & 8.75 \\
\hline $12: 45$ & 50.75 & 20.35 & 0.33 & 6.78 \\
\hline $13: 00$ & 50.94 & 20.23 & 0.30 & 6.13 \\
\hline $13: 15$ & 53.06 & 20.47 & 0.39 & 8.02 \\
\hline $13: 30$ & 54.75 & 20.97 & 0.45 & 9.44 \\
\hline $13: 45$ & 51.69 & 20.47 & 0.30 & 6.14 \\
\hline $14: 00$ & 48.44 & 20.99 & 0.46 & 9.55 \\
\hline $14: 15$ & 49.75 & 20.91 & 0.34 & 7.00 \\
\hline $14: 30$ & 48.94 & 20.67 & 0.44 & 9.14 \\
\hline $14: 45$ & 49.13 & 20.82 & 0.40 & 8.35 \\
\hline $15: 00$ & 47.88 & 20.54 & 0.39 & 8.01 \\
\hline $15: 15$ & 48.25 & 21.60 & 0.43 & 9.31 \\
\hline $15: 30$ & 47.38 & 21.83 & 0.34 & 7.44 \\
\hline $15: 45$ & 47.06 & 21.94 & 0.48 & 10.44 \\
\hline $16: 00$ & 48.31 & 21.79 & 0.32 & 6.91 \\
\hline $16: 15$ & 46.88 & 19.81 & 0.39 & 7.65 \\
\hline $16: 30$ & 46.38 & 19.48 & 0.39 & 7.60 \\
\hline $16: 45$ & 45.19 & 19.51 & 0.20 & 3.80 \\
\hline $17: 00$ & 44.81 & 18.16 & 0.30 & 5.45 \\
\hline $17: 15$ & 43.63 & 18.00 & 0.41 & 7.33 \\
\hline & & & & \\
\hline
\end{tabular}


Rickric O. Gratela et al., International Journal of Emerging Trends in Engineering Research, 8(8), August 2020, 4079 - 4083

Table 3: Output Data with Cooling System Applied to PV

Panel

\begin{tabular}{|c|c|c|c|c|}
\hline Time & Temp & Voltage & Current & Power \\
\hline $10: 00$ & 31.69 & 21.92 & 1.67 & 36.63 \\
\hline $10: 15$ & 33.94 & 21.94 & 1.56 & 34.25 \\
\hline $10: 30$ & 33.19 & 20.62 & 1.64 & 33.88 \\
\hline $10: 45$ & 32.50 & 21.75 & 1.63 & 35.43 \\
\hline $11: 00$ & 32.31 & 21.06 & 1.51 & 31.84 \\
\hline $11: 15$ & 34.25 & 21.14 & 1.40 & 29.66 \\
\hline $11: 30$ & 33.56 & 20.77 & 1.53 & 31.84 \\
\hline $11: 45$ & 31.94 & 21.07 & 1.63 & 34.28 \\
\hline $12: 00$ & 32.88 & 20.11 & 1.76 & 35.47 \\
\hline $12: 15$ & 34.56 & 21.16 & 1.43 & 31.64 \\
\hline $12: 30$ & 31.63 & 20.89 & 1.59 & 33.17 \\
\hline $12: 45$ & 35.69 & 20.60 & 1.44 & 29.71 \\
\hline $13: 00$ & 33.13 & 20.67 & 1.53 & 32.57 \\
\hline $13: 15$ & 32.81 & 20.31 & 1.62 & 32.80 \\
\hline $13: 30$ & 33.38 & 21.17 & 1.58 & 33.34 \\
\hline $13: 45$ & 31.44 & 20.65 & 1.61 & 33.16 \\
\hline $14: 00$ & 34.13 & 21.01 & 1.48 & 32.60 \\
\hline $14: 15$ & 32.31 & 21.11 & 1.74 & 36.69 \\
\hline $14: 30$ & 32.63 & 20.89 & 1.77 & 36.87 \\
\hline $14: 45$ & 31.88 & 21.08 & 1.90 & 40.03 \\
\hline $15: 00$ & 33.13 & 20.56 & 1.51 & 31.00 \\
\hline $15: 15$ & 34.44 & 21.19 & 1.47 & 31.21 \\
\hline $15: 30$ & 33.23 & 22.11 & 1.59 & 35.15 \\
\hline $15: 45$ & 34.75 & 21.87 & 1.53 & 33.35 \\
\hline $16: 00$ & 32.75 & 21.63 & 1.61 & 34.87 \\
\hline $16: 15$ & 31.19 & 19.77 & 1.61 & 31.73 \\
\hline $16: 30$ & 32.38 & 19.34 & 1.61 & 31.20 \\
\hline $16: 45$ & 31.69 & 19.37 & 1.29 & 24.97 \\
\hline $17: 00$ & 30.63 & 18.24 & 1.30 & 23.69 \\
\hline $17: 15$ & 30.31 & 17.90 & 1.30 & 23.22 \\
\hline
\end{tabular}

Figure 3 shows the comparison of the two setups by their current, temperature and voltage with the same amount of inputirradiance.

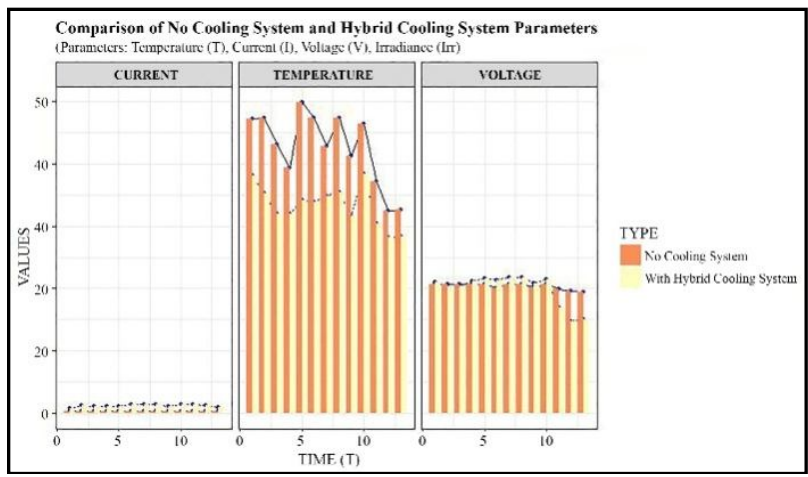

Figure 3: Comparison of Cooling and Non-Cooling Setup

Figure 4 shows the graph of temperature between setup 1 and setup 2 which has the mid difference compared to the other two parameters.

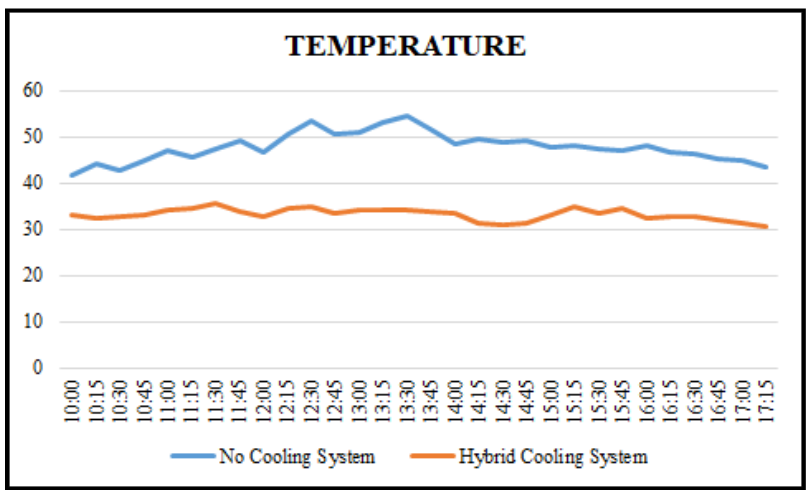

Figure 4: Temperature of Panel with Cooling and Non-cooling Mechanism

The difference shown in Figure 5 gives the graph for the current which proves the effect of the combination of air- and water-cooling mechanism has enhanced the process of maintaining low operating temperature thus, greater efficiency among all the parameters provided.

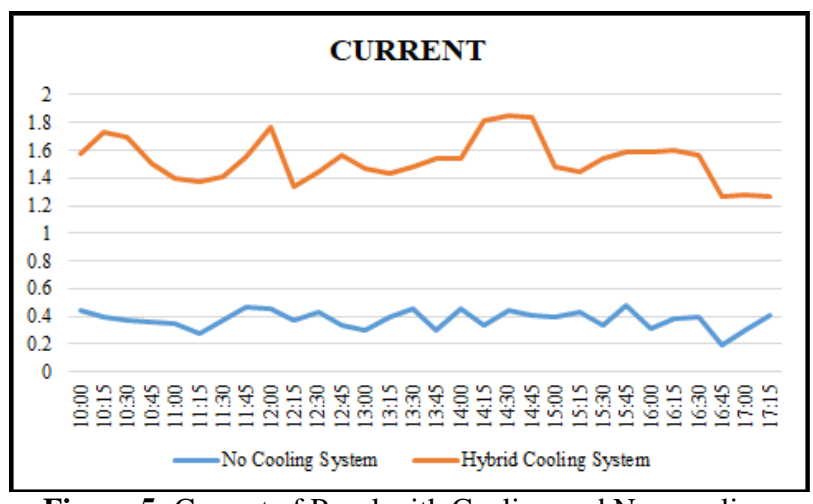

Figure 5: Current of Panel with Cooling and Non-cooling Mechanism

On the other hand, the graph of the voltage in Figure 6 has low discrepancy since the buck-boost system has a factor of sustaining the voltage linear while focusing on improving the current.

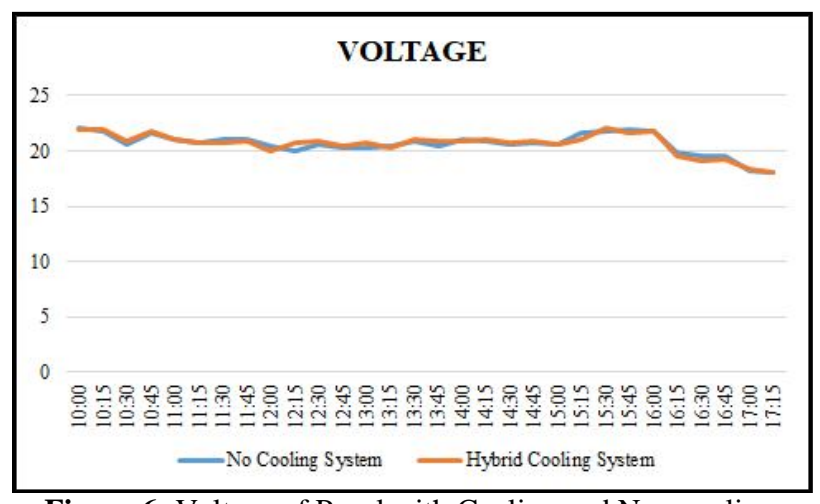

Figure 6: Voltage of Panel with Cooling and Non-cooling Mechanism 
Figure 7 clearly shows the overall result of power output by using the hybrid cooling mechanism compared to the solar panel alone. It was almost similar to the graph of the current since the voltage is held linear.

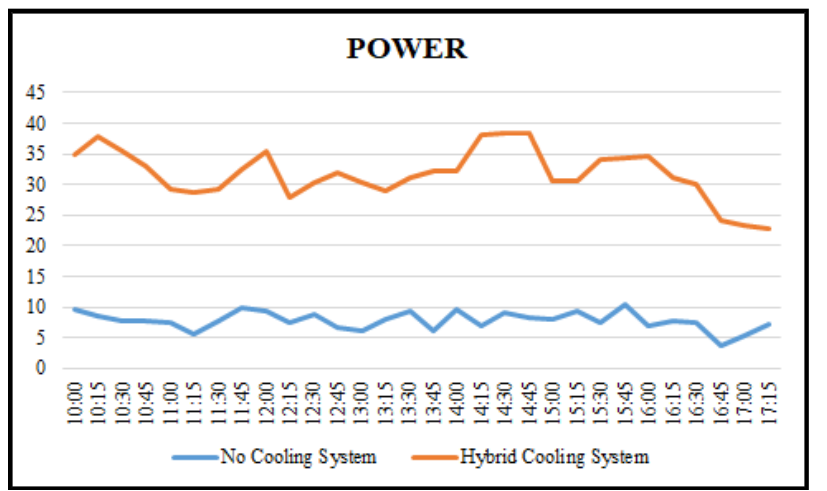

Figure 7: Power of Panel with Cooling and Non-cooling Mechanism

\section{CONCLUSION}

This research intends to develop a hybrid cooling system by using air-cooling and water-cooling fluids simultaneously unto solar PV panel's surface to avoid high temperature during operation which further leads to deficiency.

Using the results from the gathered data, it has been determined that greater irradiance with longer exposure to the direct sun provokes higher temperature but lower current output. By implementing the hybrid cooling system to the panel, the energy output became more improved.

Aside from voltage, the current, the temperature as well as the output power of the setup with hybrid cooling system proves a lot efficient compared to the other setup. The efficiency increased by $22.5 \%$ compared to the original setup without cooling system. The average electricity delivered were also boosted by the self-cleaning effect. The increase in efficiency of PV panel signifies the investment will have a shorter payback period as economic feasibility was determined and the PV panel will have a longer lifespan.

\section{ACKNOWLEDGEMENT}

To theCollege of Engineering faculty most especially, the Electronics Engineering department of the Technological University of the Philippines Manila for sharing their knowledge as well as their resources and to our parents for their endless support. Above all, God for His continuous guidance.

\section{REFERENCES}

[1] K. Vidyanandan, "An Overview of Factors Affecting the Performance of Solar PV Systems," Energy Scan, vol. 27, pp. 2-8, 2017.

[2] R. L. Ali, "Automatic Sun Tracking System," May
2005.

[3] R. Ali and S. Celik, "Effect of Cooling on Solar Panel Performance," vol. 100, 2017.

[4] S. A. Meshram, S. A. Kapade, A. D. Choudhari, R. S. Kakade, A. V. Mhala and K. B. Nagane, "Solar PV System for Electric Traction Application with Battery Backup," International Journal of Emerging Technologies in Engineering Research (IJETER), vol. 7, no. 1, 2019.

[5] F. Grubisic-Cabo, S. Nizetic and G. Tina, "Photovoltaic panels: A review of the cooling techniques," Transactions of FAMENA, vol. 40, pp. 63-74, 2016.

[6] S. Nizetic, D. Coko, A. Yadav and F. Grubisic-Cabo, "Water spray cooling technique applied on a photovoltaic panel: The performance response," Energy Conversion and Management, vol. 108, pp. 287-296, 2016. https://doi.org/10.1016/j.enconman.2015.10.079

[7] L. K. S. Tolentino, F. R. G. Cruz, R. G. Garcia and W.-Y. Chung, "Maximum Power Point Tracking Controller IC Based on Ripple Correlation Control Algorithm," in 8th IEEE International Conference Humanoid, Nanotechnology, Information Technology Communication and Control, Environment and Management (HNICEM), Cebu, Philippines, 2015.

[8] M. Yusoff, L. Waie Zhe, M. Irwanto, M. AbdulMalek, A. Amelia, G. Nair and S. Ibrahim, "Analysis air cooling mechanism for photovoltaic panel by solar simulator," International Journal of Electrical and Computer Engineering, vol. 5, pp. 636-643, 2015. https://doi.org/10.11591/ijece.v5i4.pp636-643

[9] H. H. El-Ghetany, H. M. Elgohary and Y. M. Mohammed, "Performance Evaluation and Experimental Verification of a Novel Solar Water Desalination System," International Journal of Emerging Technologies in Engineering Research (IJETER), vol. 7, no. 1, 2019.

[10] B. Du, E. Hu and M. Kolhe, "Performance analysis of water cooled concentrated photovoltaic (CPV) system," Renewable and Sustainable Energy Reviews, vol. 16, no. 9, pp. 6732-6736, 2012.

[11] H. Bahaidarah, A. Subhan, P. Gandhidasan and S. Rehman, "Performance evaluation of a PV (photovoltaic) module by back surface water cooling for hot climatic conditions," Energy, vol. 59, pp. 445-453, 2013.

https://doi.org/10.1016/j.energy.2013.07.050

[12] R. O. Gratela, J. A. S. Martes, G. I. Pagatpatan, J. P. Pagkaliwangan, D. K. A. Torcuato, T. M. Amado, A. U. Aquino, J. P. M. Ramos, E. O. Fernandez and I. C. Valenzuela, "Neuro-Fuzzy based MPPT for Solar PV Panel Hybrid Cooling System," in 2019 IEEE 11th International Conference in Humanoid, Nanotechnology, Information Technology, Communication and Control, Environment, and Management (HNICEM), 2019. 